\title{
Relation à la nature et relation pédagogique : convergences et vie éthique des enseignants
}

\section{Céline Carrey}

\section{(2) OpenEdition \\ 1 Journals}

Édition électronique

URL : http://journals.openedition.org/ere/686

DOI : $10.4000 /$ ere.686

ISSN : 2561-2271

Éditeur

Centr'ERE

\section{Édition imprimée}

Date de publication : 20 décembre 2016

ISSN : 1373-9689

\section{Référence électronique}

Céline Carrey, « Relation à la nature et relation pédagogique : convergences et vie éthique des enseignants », Éducation relative à l'environnement [En ligne], Volume 13 - 2 | 2016, mis en ligne le 20 décembre 2016, consulté le 21 février 2020. URL : http://journals.openedition.org/ere/686 ; DOI : $10.4000 /$ ere. 686 


\title{
Relation à la nature et relation pédagogique : convergences et vie éthique des enseignants
}

\author{
Céline Carrey
}

Quel enseignant ne s'est jamais interrogé sur le sens de son agir quotidien, sur les finalités de l'éducation qu'il met en œuvre? En ce qui me concerne, ceci a été accompagné d'une prise de conscience : celle que nous vivons un kairos, un seuil, un moment charnière pour notre société. En effet, de nombreux questionnements assaillent la société contemporaine, remettant en question les valeurs qui la fondent et ses grands principes de fonctionnement. Penser la transition devient alors un impératif. Or depuis Émile Durkheim, on reconnait que toute éducation est un fait social indéniablement lié à son époque et ancré dans l'histoire de l'humanité, mais audelà, il semble aujourd'hui évident qu'«Éduquer, c'est faire un pari sur l'avenir » (Hannoun, 1998). S'interroger sur les finalités éducatives, c'est donc s'interroger sur l'être humain, mais également sur la société à venir. Ainsi, dans le contexte actuel d'une crise environnementale sans précédent, j'ai fait mienne cette double interrogation rappelée par Pierre Rabhi (2010) : «Quelle planète laisserons-nous à nos enfants ? » et « Quels enfants laisserons-nous à notre planète ? ».

C'est dans les racines philosophiques de l'éducation d'une part, et de la relation à la nature d'autre part, que je suis allée puiser pour mieux comprendre leurs fondements et les liens qui les unissent. Je tenterai donc ici de partager mes découvertes, en commençant par exposer comment ces deux types de relations - relation à la nature et relation pédagogique - semblent indissociables et éthiquement liés. Puis une analyse de chacune montrera des similitudes de classement possibles, autour des trois théories morales qui fondent, selon Didier Moreau, la pensée occidentale. Enfin, nous verrons comment relation pédagogique et relation à la nature peuvent s'enrichir mutuellement, et alimenter ainsi la construction d'une vie éthique des enseignants au sein de ce contexte de kairos. 


\section{Relation à la nature et relation pédagogique : une origine éthique commune.}

Si l'on s'intéresse à l'origine de l'éthique au sein de la tradition philosophique occidentale, Socrate y est reconnu comme un fondateur de cette question. Pour Didier Moreau, la question socratique se résume à celle-ci : «Comment doit-on vivre?» (Moreau, 2011). Cela implique tout d'abord des questionnements sur la connaissance de ce qui nous entoure et la manière d'accéder à cette connaissance. Par ailleurs, , il s'agit directement de la connaissance de soi, d'où la reprise par Socrate de la maxime gravée sur le temple d'Apollon à Delphes: "Connais-toi toi-même». Or cela suppose précisément que l'on ne sache pas, de façon innée, comment il nous faut vivre. Cela fait appel à 'un apprentissage, une éducation. C'est pourquoi, selon Didier Moreau (2011), éducation et éthique sont intrinsèquement liées: toute éducation est éthique, mais également toute éthique implique l'éducation d'autrui et de soi-même. L'éthique ne serait pas un "surplus » que l'éducateur doit posséder. Elle est fondamentalement » organiquement » nous dit-il - incluse dans l'acte éducatif.

Au-delà d'une connaissance sur ce qui nous est extérieur et sur notre intérieur, la question socratique paraît également nous interroger sur la relation entre intérieur et extérieur, sur ce qui les relie. Ainsi, dans le courant de la phénoménologie, la recherche ontologique est indissociable de la considération de notre "être-au-monde». Dans l'héritage de cette pensée, Dominique Cottereau précise :

Cette expression " être-au-monde " permet de réaliser que l'être n'est rien sans le monde dans lequel il vit, et que le monde est bien composé de l'ensemble des êtres qui le peuplent. Se pencher sur l'être-au-monde c'est entrer dans ce qui forme la relation de chacun à son entourage. (Cottereau, 1999, p.10, citée par Beaudout, 2009, p. 260)

Or qu'est-ce que le monde qui nous entoure ? De qui, de quoi s'agit-il ? L'éthique doitelle restreindre son champ d'action à nos relations sociales avec les autres humains comme elle l'a majoritairement fait dans la tradition philosophique occidentale ? Ou bien faut-il (ré)intégrer au sein de notre " monde », et de nos considérations éthiques, tous les êtres vivants et entités qui nous entourent? Cette dernière option implique un changement de paradigme afin d'aborder sous un jour nouveau la question de l'éthique dans notre relation à la nature. Ainsi selon le philosophe Hicham-Stéphane Afeissa,

l'une des convictions que partagent bon nombre d'éthiciens de l'environnement est qu'il est nécessaire d'interroger explicitement les modalités générales du rapport à la nature tel qu'il a été pensé par la tradition philosophique, morale, scientifique et religieuse occidentale, si bien qu'il ne nous soit plus possible de puiser sereinement dans cette tradition les éléments permettant de fournir une solution aux problèmes écologiques auxquels nous sommes confrontés, dans la mesure où cette tradition fait elle-même partie du problème. (Afeissa, 2013, p. 188-189)

Certaines éthiques environnementales d'origine anglo-américaine semblent, sur ce point, offrir une alternative à la pensée de la philosophie de la nature fondée dans la métaphysique européenne.Ici, l'enjeu principal semble concerner la reconnaissance ou non des entités de la nature - ou une partie de celles-ci - en tant que patients moraux. Selon Gérald Hess un patient moral est «celui envers qui un agent [moral] est moralement obligé » (Hess, 2013, p. 88) et ce qui rend l'agent moral obligé ou non vis-àvis de l'entité, c'est la valeur de celle-ci ${ }^{1}$. Cependant tout le débat en éthique environnementale porte justement sur quelles entités naturelles sont intégrées au sein 
de la communauté morale, et quelle valeur intrinsèque leur est reconnue. Gérald Hess propose dans le même ouvrage un classement des différentes éthiques environnementales, dont voici un résumé très succinct :

- Théocentrisme : la moralité et la valeur des entités sont issues du Divin.

- Anthropocentrisme : seul l'homme est reconnu comme patient moral.

- Pathocentrisme: la valeur des entités naturelles dépend de leur capacité à ressentir (en débat pour certains animaux et les plantes).

- Biocentrisme : le critère étant celui de la vie, tout être vivant devient patient moral.

- Écocentrisme (I) : L'ensemble de la biosphère et toutes ses entités organiques et non organiques, ainsi que les écosystèmes, sont reconnus patients moraux.

- Écocentrisme (II) - Holisme : Gérald Hess propose de séparer ici les éthiques holistes, bien qu'écocentriques, parce qu'elles ne sont pas extensionnistes, c'est-à-dire non issues d'un anthropocentrisme étendu à d'autres entités. Elles ne sont pas basées sur une séparation initiale entre l'humain et la nature, mais sur une appartenance commune à un tout unitaire.

\section{Trois statuts de la nature}

Ce qui retient particulièrement mon attention dans les propos de Gérald Hess, c'est l'analyse qu'il présente des différents statuts attribués à la nature au sein de l'éthique environnementale, car selon lui, la nature est tout d'abord représentation. Ainsi il en distingue trois: nature artefact, nature poïésis, nature habitat. À chacune, je tenterai d'associer ici certaines éthiques environnementales et les considérations qui en découlent, selon ma propre interprétation.

La nature artefact correspond selon Gérald Hess, à une approche de type prométhéen, basée sur la rupture entre l'humain et la nature et une relation de domination/ soumission. Les éthiques environnementales anthropocentriques sont basées sur cette représentation, l'espèce humaine étant considérée comme supérieure aux autres espèces. Dans le cas de l'anthropocentrisme élargi aux grands singes, ou des éthiques pathocentriques, on reconnaîtra à ces dernières, un certain élargissement du registre des valeurs. Mais dans tous les cas, il s'agit d'une perspective majoritairement technoscientifique: les entités naturelles sont essentiellement considérées comme des ressources, objets soumis à la domination ou à la gestion par les humains. La valeur attribuée aux entités naturelles dépend majoritairement de leur lien avec l'espèce humaine, et elles ne sont souvent pas reconnues comme patients moraux à part entière. Ceci correspond à la représentation dominante de la nature au sein de la société occidentale. Le christianisme, relayé ensuite par la science et la technique, a longtemps fait la promotion d'une représentation d'une nature soumise, au service de l'être humain. Cependant l'interprétation des textes bibliques fait débat ; en effet, la dernière encyclique du pape (François $1^{\mathrm{er}}$, 2015) marque une rupture officielle, puisqu'elle est une invitation à un changement de représentation de la nature et de la relation entretenue avec celle-ci.

Deuxième représentation selon Gérald Hess, la nature poḯsis est basée sur la pensée d'Aristote selon laquelle toute entité naturelle n'est pas le résultat d'une action extérieure, mais plutôt une production d'elle-même. Cette représentation reconnaît donc une nature "autonome, vivante, agissante par elle-même " (Hess, 2013, p. 49), selon une perspective génétique. Les éthiques biocentristes semblent inspirées de cette représentation: une valeur propre y est attribuée à tous les êtres vivants, avec 
reconnaissance d'une certaine finalité qui les rapproche des conceptions vitalistes. De même, les éthiques écocentristes (I) basées sur des considérations égalitaires (toutes les entités vivantes possèdent la même valeur) malgré le problème de l'antagonisme entre les intérêts humains et ceux du vivant en général. Pour tenter d'y échapper, certains auteurs prônent l'idée d'une hiérarchisation des valeurs, ce qui entraîne le problème du choix des critères de hiérarchisation, rapprochant alors ces propositions d'un anthropocentrisme étendu.

La nature habitat est le troisième type de représentation distingué par Gérald Hess. En s'appuyant sur la racine étymologique du préfixe «éco "-oikos, l'habitat en greccette approche valorise la nature vécue comme lieu d'existence de l'être humain. On y retrouve les éthiques écocentristes de type holistique, lesquelles considèrent l'homme et l'ensemble du vivant comme appartenant à un Tout. La notion d'interdépendance y est fondamentale, et l'on peut considérer que cette approche est surtout basée sur la reconnaissance et la valorisation de la relation que l'humain entretient avec le monde qui l'entoure. La perspective y est donc majoritairement phénoménologique. Cette approche propose une alternative à la vision dualiste opposant nature et culture qui caractérise la représentation de la nature artefact. Cependant, elle ne résout pas l'antagonisme existant entre les intérêts humains et ceux du vivant en général, et dans certains cas, engendre un débat autour de l'idée d'un «bien » qui serait naturel, se rapprochant de certaines formes spirituelles transcendantales.

Cet essai de classification met en évidence trois statuts principaux attribués à la nature : artefact, poïésis et habitat, correspondant chacun à une modalité spécifique de relation à la nature : de domination, égalitaire et d'interdépendance. Mais qu'en est-il des statuts de l'apprenant au sein de la relation pédagogique?

\section{Trois postures pédagogiques}

Étymologiquement, éduquer provient d'une part de educare qui signifie nourrir, entretenir, élever et porte donc l'idée de prendre soin, et d'autre part de educere qui évoque l'idée d'un passage d'un état à un autre, d'une transformation. S'agit-il donc de prendre soin, d'accompagner ou de former, de transformer? Les différentes définitions existantes des notions d'éducation, d'enseignement, d'instruction et de formation sont autant de preuves de finalités différentes et des multiples facteurs influençant l'acte éducatif. Le statut de l'apprenant fait également historiquement débat : homme social, naturellement bon pour Rousseau qui prône une "éducation négative", ou au contraire, asocial pour Durkheim, tabula rasa à qui il faut donner forme. L'enseignant doit trouver sa place au sein d'une relation qui, comme le fait remarquer Gaston Pineau (2002), est souvent modélisée de façon ternaire.

Ainsi, dans le triangle de Jean Houssaye (1988) la relation entre le maitre, l'élève et le savoir peut être celle de former, enseigner et apprendre, alors que l'Institut Français de l'Éducation ${ }^{2}$ proposera les postures de contrôle, lâcher-prise et accompagnement. Philippe Meirieu (2003) présente quant à lui, dans un rapport élaboré pour l'UNESCO, les postures du clerc, du compagnon et du bibliothécaire. Les classifications sont nombreuses et se complètent. Pour la suite de cette étude, j'ai choisi de me baser sur les travaux de Frédérique Lerbet-Séréni qui dégage trois postures de l'accompagnant en éducation, inspirées des différentes postures du "guide» de montagne: guide, compagnon et accompagnateur (Lerbet-Séréni, 2003). Cette catégorisation, qui montre 
bien l'intérêt de considérer la relation à la nature pour penser l'éthique éducative, a le mérite de s'appuyer sur des représentations imagées, faciles à saisir au premier abord. En reprenant la terminologie de cette chercheure, je vais tenter de caractériser les différentes postures, tout en les complétant par une interprétation personnelle contenant des références aux autres classifications citées ci-dessus, puis d'en proposer une synthèse.

Le guide: cette posture, pour F. Lerbet-Séréni, est inspirée du guide de haute montagne, qui face au danger est celui qui ouvre le chemin, les autres étant soumis à son autorité et devant agir par mimétisme. Dans cette posture pédagogique, le guide est celui qui a le contrôle absolu, qui détient le savoir, il décide de la pédagogie, des rythmes, des contenus. Cette posture semble correspondre à la pédagogie traditionnelle, que Philippe Meirieu nomme du «clerc » et qui est issue de la méthode des Frères des écoles chrétiennes dont on s'est inspirée dans les premières écoles normales. Le type de relation au sein du triangle de Houssaye semble être celui reliant le maître au savoir: enseigner. L'égalité maître-élève devient ici le but final de l'éducation avec pour objectif l'émancipation de l'individu, héritage de la pensée des Lumières.

Le compagnon: Cette posture évoque un cheminement commun en montagne, sans danger particulier. Pédagogiquement ceci implique dès le départ, la reconnaissance d'une égalité entre l'enseignant et l'apprenant. Il s'agit ici d'apprendre ensemble, en ayant recours à un certain lâcher-prise. On y retrouve le concept de l'apprenant "naturellement bon" issu de la pensée Rousseauiste, et sur lequel il faut tenter de minimiser son influence, mais également le principe « d'égalité des intelligences » dans Le maître ignorant (Rancière, 1987). Le savoir est, dans ce cas, extérieur à l'accompagnant. La relation privilégiée dans le triangle de Houssaye semble être ici celle reliant les élèves au savoir : apprendre. Cette posture évoque plusieurs tendances allant de la non-directivité de Carl Rogers appliquée en autogestion pédagogique, jusqu'à des postures plus modérées que l'on retrouve dans les pédagogies de l'école nouvelle.

L'accompagnateur : s'appliquant davantage à la moyenne montagne, où les risques sont modérés, l'accompagnateur est à l'arrière et s'assure de l'absence de danger et que tout va bien pour tous les membres du groupe. Il s'agit d'une posture portant une asymétrie assumée, mais où la réciprocité n'est pas exclue. En terme pédagogique, l'accompagnateur possède un certain savoir, pouvant être incomplet, et il va porter son attention sur ce que l'autre vit, découvre, en essayant de lui apporter son soutien en fonction de ses besoins. L'apprenant y est reconnu singulier et capable d'autonomie. Cette posture fonde l'idée d'une gestion de l'hétérogénéité au sein de la classe, d'un enseignant qui accompagne les élèves sur le chemin de l'apprentissage, en les écoutant et en s'adaptant à leur singularité. Pour Frédérique Lerbet-Séréni (2014), l'accompagnement implique que «quelqu'un doit passer par quelqu'un d'autre que lui pour se produire lui-même ». Dans le triangle de Houssaye il s'agit bien d'une relation privilégiée entre maître et élève: former. Mais la posture d'accompagnateur de Frédérique Lerbet-Séréni me semble évoquer surtout la pensée de l'autoformation selon l'approche herméneutique de Gaston Pineau, pour qui :

Se former, se donner une forme, est une activité plus ontologique que s'éduquer, c'est-à-dire s'élever, se nourrir. Se former, c'est reconnaître qu'aucune forme achevée n'existe a priori qui serait donnée de l'extérieur. Cette forme toujours 
inachevée dépend d'une action. Sa construction propre est une activité

permanente. (Pineau, 1994)

Pour Frédérique Lerbet-Séréni, c'est dans un équilibre entre ces trois postures que se situerait une posture idéale. Sans vouloir considérer chacune d'entre elles comme un absolu, nous nous appuierons sur cette classification pour établir des liens avec le champ des éthiques environnementales. Cependant, nous allons voir qu'il semble difficile de penser à un équilibre entre les trois, chacune pouvant être rapprochée d'une théorie morale différente, correspondant à une éthique bien distincte.

\section{Convergences}

Tant en ce qui concerne les différents statuts de la nature que les diverses postures pédagogiques, , la classification dépend du statut que l'on attribue à l'Autre (apprenant ou entité naturelle), et donc du « Comment doit-on vivre ? » vis-à-vis de cet Autre : cela fait référence à différents choix éthiques. Or selon Didier Moreau (2011), si les éthiques sont nombreuses, les théories morales auxquelles elles peuvent être rattachées sont en nombre limité. Ce philosophe en distingue trois qui correspondent à trois épistémès fondamentales de la pensée occidentale : platonisme, scepticisme, et stoïcisme.

La première de ces théories morales prend son origine dans le Mythe de la Caverne où Platon nous présente une conversion du regard - des étants aux Idées - qui mène à la vérité. Il introduit donc une rupture entre le monde sensible des étants, imparfait, et le monde des Idées, suprasensible, qui seul permet l'accès à la vérité et la connaissance de l'essence des choses. La nature appartient au monde sensible, et c'est en s'en détachant, en détournant notre regard des ombres dans la caverne, que nous pouvons briser nos chaînes et accéder à la connaissance de la vérité du monde des Idées : voilà ce qui fonde l'idée du Bien. L'essence de l'homme est donc de dépasser sa nature par une conversion nécessaire, afin d'accéder à cette vérité transcendantale, l'» Idée des Idées ». L'épistémè platonicienne est en ce sens fondatrice de l'idée de rupture entre l'homme et la nature. Elle sera reprise par le catholicisme de Saint-Augustin qui renforcera l'idée de rupture, en désignant une nature corrompue et dangereuse, et introduira le Divin du monde suprasensible comme origine de toute conversion. Puis sur cette base, Descartes fondera le dualisme caractéristique de la pensée scientifique occidentale, le monde sensible devenant objet sous l'influence de la pensée mécaniciste. Il s'agit bien ici d'une représentation d'une nature artefact. L'éducation occidentale traditionnelle est, quant à elle, tout à fait liée à l'épistémè platonicienne. En effet, elle prend son origine chez les Frères des écoles chrétiennes qui se sont inspirés, nous l'avons vu, de la vision platonicienne de la conversion. Par la suite, le mouvement des Lumières, fondateur de l'éducation encyclopédique universelle, substituera Dieu par la Raison comme origine de toute conversion devant mener à l'émancipation du citoyen.

Le scepticisme, deuxième épistémè représentée notamment par le philosophe Pyrrhon, consiste à considérer qu'on ne peut atteindre la vérité avec certitude. Ainsi l'accès à la connaissance est impossible et il faut sans cesse intégrer la notion de doute dans la recherche. Cette théorie morale donnera naissance au relativisme moral. En effet, il semble difficile de poser une éthique basée sur le scepticisme, puisque le bien ne peut pas être pensé ni appréhendé par l'homme. La seule issue possible serait donc seulement de fuir le mal, d'éviter les actes dont les conséquences sont négatives, mais sans espoir de pouvoir ni progresser ni se convertir en un homme meilleur. David 
Hume, philosophe sceptique, considère alors que l'on ne peut s'en tenir qu'à notre perception sensorielle $\mathrm{du}$ monde, toute interprétation métaphysique étant immédiatement mise en doute par le scepticisme. Le scepticisme remet donc en cause l'idée d'une réalité transcendante, et ne peut que s'appuyer sur une approche probabilistique permettant d'émettre des hypothèses. Dans le champ des éthiques environnementales, on peut supposer que le scepticisme ne permet pas de déterminer la valeur des différentes entités naturelles, ou de les hiérarchiser puisqu'aucune vérité n'est atteignable. Ainsi la perception du monde ne peut qu'engendrer la représentation d'une nature poïésis attribuant une égalité de valeurs aux différentes entités. En ce qui concerne le domaine éducatif, une éthique basée sur le scepticisme ne peut qu'engendrer une posture basée sur la non-directivité, la reconnaissance de l'autre comme un égal, et une posture pédagogique qui ne peut correspondre qu'à celle du compagnon, où l'influence de l'éducateur est la plus réduite.

Le stoïcisme quant à lui, est basé sur le concept d'unité et d'appartenance. Dans le stoïcisme, pas de réalité autre derrière les étants ; ce qui est, c'est ce qu'on appréhende, et tout fait partie d'une unité : le Cosmos. Ceci évoque une vision holistique de la relation avec la nature : l'homme appartient à ce cosmos, et il doit y trouver sa place. Nous renouons ici avec le questionnement socratique perçu dans sa dimension relationnelle avec le monde qui nous entoure. La théorie morale fondée par le stoïcisme est basée sur le libre arbitre dans la recherche d'un équilibre entre tendance naturelle (hormé) et raison (logos) $)^{3}$ et valorise autant l'excellence des moyens mis en œuvre (skopos) que la correspondance entre l'objectif initial et le résultat final (telos). Le sage est une figure d'exception qui fait référence; les autres, selon le stoïcisme impérial de Marc Aurèle, sont des aspirants à la sagesse, des proficiens, qui cheminent dans un perfectionnement perpétuel grâce à leurs actions dans le monde. C'est ce qui permet à Didier Moreau d'affirmer qu'il s'agit d'une éthique appliquée, qui génère de nouvelles normes et participe à la construction d'une vérité et d'une éthique jamais achevées. Selon cette perspective, l'éducation des néoï - les nouveaux venus - est donc métamorphose permanente, recherche d'un perfectionnement éthique perpétuel, sans vérité transcendante. Ceci n'est pas sans évoquer la vision de l'humanisme de la Bildung de Wilheim von Humboldt inspirant le paradigme de l'éducation tout au long de la vie ${ }^{4}$ : la Bildung est un déploiement de soi fondé sur la libre individualité, la richesse de la singularité, la recherche d'une sagesse tenant compte de la totalité de l'humain en formation.

Après ces efforts de catégorisation, nous pouvons mettre en parallèle les trois analyses et tenter d'établir des liens entre chaque épistémè et les catégories de relation à la nature et de relation pédagogique. Le tableau 1 présente cette synthèse.

Tableau 1 : Convergences éthiques de la relation à la nature et de la relation pédagogique autour des trois épistémès de référence.

\begin{tabular}{|l|l|l|l|}
\hline & Épistémè de référence & Relation à la nature & Relation pédagogique \\
\hline
\end{tabular}




\begin{tabular}{|c|c|c|c|}
\hline \multirow{3}{*}{$\begin{array}{l}\text { Platonisme } \\
\text { Théorie de la } \\
\text { conversion }\end{array}$} & $\begin{array}{l}\text { Statut : } \\
\text { Entité/individu à } \\
\text { (trans)former } \\
\text { Valeur attribuée par } \\
\text { rapport à un idéal } \\
\text { étalon }\end{array}$ & $\begin{array}{l}\text { Nature artefact } \\
\text { Étalon de valeur : l'homme. }\end{array}$ & $\begin{array}{l}\text { Apprenant tabula rasa } \\
\text { Étalon de valeur: } \\
\text { l'adulte. }\end{array}$ \\
\hline & $\begin{array}{l}\text { Type de relation : } \\
\text { Rapport } \\
\text { domination/ } \\
\text { soumission. }\end{array}$ & $\begin{array}{l}\text { Rupture homme/nature } \\
\text { Domination de l'homme sur } \\
\text { la nature la transformant } \\
\text { selon une vision } \\
\text { prométhéenne et techno- } \\
\text { scientifique utilitaire }\end{array}$ & $\begin{array}{l}\text { Relation pédagogique } \\
\text { fortement asymétrique } \\
\text { et unidirectionnelle } \\
\text { L'enseignant a la } \\
\text { maîtrise de la situation } \\
\text { et possède le savoir. Il } \\
\text { transmet son savoir pour } \\
\text { former le futur adulte. }\end{array}$ \\
\hline & $\begin{array}{l}\text { Postures éthiques : } \\
\text { Éthique kantienne; } \\
\text { éthiques utilitaristes et } \\
\text { conséquentialistes. }\end{array}$ & $\begin{array}{l}\text { Anthropocentrisme. } \\
\text { Pathocentrisme } \\
\text { (anthropocentrisme étendu) }\end{array}$ & $\begin{array}{l}\text { Guide, clerc } \\
\text { Pédagogie traditionnelle }\end{array}$ \\
\hline \multirow{3}{*}{$\begin{array}{l}\text { Scepticisme } \\
\text { Théorie du } \\
\text { relativisme }\end{array}$} & $\begin{array}{l}\text { Statut: } \\
\text { Entité/individu créatif } \\
\text { et autonome } \\
\text { Valeur propre, absence } \\
\text { d'idéal étalon }\end{array}$ & $\begin{array}{l}\text { Nature poïsis } \\
\text { Absence d'étalon }\end{array}$ & $\begin{array}{l}\text { Apprenant } \\
\text { « naturellement bon » } \\
\text { Absence d'étalon }\end{array}$ \\
\hline & $\begin{array}{l}\text { Type de relation : } \\
\text { Rapport égalitaire }\end{array}$ & $\begin{array}{l}\text { Relation égalitaire selon une } \\
\text { perspective génétique }\end{array}$ & Relation symétrique \\
\hline & $\begin{array}{l}\text { Éthiques, postures : } \\
\text { Éthiques de la } \\
\text { discussion, de la justice, } \\
\text { de la vertu } \\
\text { Éthique de la sollicitude } \\
\text { (care) }\end{array}$ & $\begin{array}{l}\text { Biocentrisme. } \\
\text { Écocentrisme (I) }\end{array}$ & $\begin{array}{l}\text { Compagnon. } \\
\text { Pédagogie non directive, } \\
\text { autogestion } \\
\text { pédagogique, école } \\
\text { nouvelle }\end{array}$ \\
\hline $\begin{array}{l}\text { Stoïcisme } \\
\text { Théorie de la } \\
\text { métamorphose }\end{array}$ & $\begin{array}{l}\text { Statut: } \\
\text { Entité/individu } \\
\text { singulier et portant une } \\
\text { part de mystère } \\
\text { Idéal défini dans un } \\
\text { équilibre au sein d'une } \\
\text { appartenance } \\
\text { commune (holisme) }\end{array}$ & $\begin{array}{l}\text { Nature habitat } \\
\text { Idéal de symbiose homme/ } \\
\text { nature }\end{array}$ & $\begin{array}{l}\text { Apprenant néoïs, autre } \\
\text { singulier. } \\
\text { Idéal d'équilibre entre } \\
\text { hormé et logos. }\end{array}$ \\
\hline
\end{tabular}




\begin{tabular}{|l|l|l|}
\hline $\begin{array}{l}\text { Type de relation : } \\
\text { Rapport de reliance }\end{array}$ & $\begin{array}{l}\text { Appartenance commune à } \\
\text { un Cosmos sein d'une perspective } \\
\text { Relation d'interdépendance, } \\
\text { phénoménologique et } \\
\text { herméneutique }\end{array}$ & $\begin{array}{l}\text { Appartenance commune } \\
\text { l'Humanité } \\
\text { Relation légèrement } \\
\text { asymétrique, mais avec } \\
\text { possibilité de réciprocité. } \\
\text { Écoute mutuelle }\end{array}$ \\
\hline $\begin{array}{l}\text { Éthiques, postures : } \\
\text { Éthiques } \\
\text { herméneutiques, de }\end{array}$ & $\begin{array}{l}\text { l'Altérité } \\
\text { Éthiques relationnelles: } \\
\text { du lien, de la reliance, } \\
\text { de la sollicitude. }\end{array}$ & $\begin{array}{l}\text { Accompagnateur } \\
\text { Pédagogie relationnelle } \\
\text { Autoformation Éducation } \\
\text { tout au long de la vie }\end{array}$ \\
\hline
\end{tabular}

\section{Interprétation éthique}

En présentant cette synthèse, je tenterai d'énoncer rapidement les éthiques auxquelles peuvent se référer chacune des trois catégories présentées. Il est bien évidemment impossible de mener une analyse exhaustive et approfondie dans le cadre de cet article; l'objectif poursuivi est donc bien celui d'une classification éthique générale permettant une réflexion globale. Les références à des penseurs et philosophes pourront guider le lecteur s'il souhaite approfondir l'analyse amorcée.

On remarquera tout d'abord que la première catégorie se réfère systématiquement à un étalon : dans le domaine environnemental, l'humain sert de référence pour définir (par défaut) les autres entités; dans le domaine éducatif, l'enfant est considéré comme un adulte en devenir, lequel fait office de référence ${ }^{5}$. Cette pensée est profondément déterministe, puisque l'on connaît d'avance le résultat de nos actions, l'homme possède la maîtrise de l'action et de ses conséquences. Dans le cadre de cette épistémè, l'Autre est surtout considéré comme un "objet » sur lequel on agit grâce à des méthodes et techniques; dans le domaine éducatif, il s'agira de donner une place prépondérante à la didactique. Dans les deux domaines, la relation est majoritairement unidirectionnelle : l'homme agit sur la nature, l'enseignant agit sur l'apprenant. C'est une relation dominée par la rationalité, où l'on recherche l'efficacité maximale dans l'obtention d'un but bien défini. Cette approche techno-scientifique déterministe nous permet de faire le lien dans le domaine de l'éthique générale avec Kant, puisque la morale kantienne est basée sur une connaissance a priori des conséquences de nos actes, permettant ainsi une délibération morale préalable. Les éthiques utilitaristes et conséquentialistes qui en découlent (voir les propositions de Jeremy Bentham et de John Stuart Mill ) sont une adaptation pragmatique de cette pensée à la modernité. Cette catégorie, nous l'avons déjà dit, correspond majoritairement à la pensée traditionnelle, tant dans le domaine de la relation à l'environnement, qu'en éducation. Or il semble évident, dans un contexte de kairos, qu'il est nécessaire de faire évoluer nos façons de penser, et donc de renouveler nos fondements éthiques.

La deuxième catégorie est basée sur une reconnaissance égalitaire de l'Autre. Celui-ci se développe dans une auto-poïésis - autocréation, et autoformation de soi-même- 
laquelle peut évoquer la possibilité d'une certaine finalité prédéfinie (téléologie) sousjacente, mais totalement inaccessible à notre entendement. Toute valeur devient alors relative, et dans le domaine éducatif, il est impossible d'imposer à l'apprenant une direction précise puisque personne ne possède la vérité, tout comme il est impossible de hiérarchiser la valeur des différentes entités naturelles. L'éthique ne peut alors être guidée que par une recherche d'un vivre ensemble établi pour la meilleure harmonie possible. On pourra alors se référer en éthique générale, à un contractualisme, basé sur la discussion (comme chez Jürgen Habermas et et Karl-Otto Apel), sur la Justice (John Rawls), ou bien par une vertu dans le cadre d'une téléologie aristotélicienne ou rénovée selon Alasdair MacIntyre. Mais l'on pourra également s'intéresser dans cette catégorie, aux éthiques de la reconnaissance et de la sollicitude (care), puisqu'il s'agit bien d'une demande de reconnaissance d'un statut d'égalité de la part d'un Autre, historiquement dominé. Cette deuxième catégorie semble correspondre assez bien à la pensée critique de la postmodernité. Elle s'est en effet caractérisée par une remise en cause de la pensée moderne déterministe, et a porté des revendications d'égalitarisme. Cependant, il semble évident que cet égalitarisme, tant en éducation, qu'en éthique environnementale, est difficile à assumer pleinement, et porte ses propres contradictions.

Enfin la troisième catégorie reconnaît la singularité de l'Autre et sa part de mystère (nature ou apprenant), et en cela, elle fait plutôt référence à des éthiques herméneutiques (comme chez Willard Van Orman Quine, Hans-Georg Gadamer et Paul Ricoeur). Il faut ainsi noter que les auteurs des éthiques environnementales holistes (Arne Næss, Val Plumwood et Vandana Shiva, par exemple) sont très critiques vis-à-vis de l'écologie traditionnelle, et en appellent à repenser, non seulement éthiquement, mais également ontologiquement, la relation à la nature. C'est donc bien à un changement de statut des entités naturelles qu'ils font réfèrence. Quant à l'éducation dans cette catégorie, elle ne peut être conversion uniformisante, son résultat est inconnu a priori, c'est une ouverture sur des possibles. Elle est davantage une métamorphose basée sur le relationnel : relation à soi, aux autres et aux choses. Nous retrouvons ici la conception tripolaire de la formation : selon Gaston Pineau (1985) qui s'inspire de Jean-Jacques Rousseau, elle se décline en autoformation (par Soi), hétéroformation (par les autres) et écoformation (par les choses) au sein du paradigme d'une éducation tout au long de la vie. Ceci évoque également les éthiques de l'altérité (comme chez Emmanuel Levinas et Jacques Derrida) ainsi que toutes les éthiques que je qualifierai de relationnelles : éthique du lien, de la reliance et du care. Je présenterai ici davantage ces éthiques relationnelles puisqu'il s'agit, à ma connaissance, d'éthiques relativement nouvelles pour la pensée occidentale, lesquelles, dans le cadre d'une nouvelle éthique à penser, me semblent tout à fait adaptées.

Christiane Gohier propose, dans le cadre éducatif, une éthique du lien, reposant sur l'anthropologie du don. L'acte pédagogique y est davantage considéré comme un geste supposant la réciprocité dans l'interpellation et le respect (Gohier, 2007, p. 89-90). D'autre part, selon Marcel Bolle de Bal, père de cette notion, la reliance est « l'acte de relier ou de se relier» ou «le résultat de cet acte» (Bolle de Bal, 2003, p. 103). Or, la notion de reliance, au départ réservée au domaine social, s'est élargie: on parle aujourd'hui de reliance sociale (aux autres), mais également psychologique (à soi), cosmique (au monde) et enfin de reliance cognitive, liée au paradigme de la complexité. On retrouve notamment cette notion dans les travaux de Gaston Pineau et d'Edgar Morin, ce dernier appelant même à une éthique de la reliance (Bolle de Bal, 2009). En 
effet la modernité pourrait être caractérisée par un phénomène majoritaire de déliance . Faire appel à une éthique de reliance consiste à rechercher un équilibre entre une déliance permettant l'autonomie, et une reliance assurant une certaine appartenance, un lien avec le monde qui nous entoure.

\section{La vie éthique de l'enseignant : une complexité assumée}

Bien évidemment, toute catégorisation est réductrice, et nous admettrons que c'est dans la complexité que nous pouvons appréhender le réel. Cependant, il paraît maintenant évident qu'il existe des analogies entre le statut donné à la nature et celui donné à l'apprenant, lesquels correspondent à des postures éthiques spécifiques, reliées elles-mêmes à des épistémès de référence. C'est en s'appuyant sur le concept de vie éthique de Bernard Williams repris par Didier Moreau, que nous pourrons mieux saisir l'importance des apports de l'analyse menée jusque-là.

Pour Didier Moreau l'acte d'éduquer est un acte éthique en soi. C'est cela qui donne à l'éducateur la possibilité de devenir un agent moral et c'est en tant qu'agent moral qu'il peut construire une éthique liée à sa praxis. En effet, dans le domaine éducatif, il n'est plus possible aujourd'hui de penser à des délibérations morales a priori de l'acte éducatif (et donc de se baser sur une éthique kantienne), et l'enseignant ne peut recourir qu'à une éthique appliquée où chaque acte moral dépend, non de convictions préétablies, mais plutôt d'une prise de décision contextualisée, correspondant à un conflit de valeurs, spécifique à chaque cas. Or, selon Bernard Williams, le sentiment est fondateur de l'éthique, et en particulier celui qu'il nomme le regret d'agent tel que Didier Moreau le définit :

Le regret d'agent est l'émotion morale qui nous fait basculer de la rationalité, qui nous assure que nous ne sommes pas responsables et n'avons pas à nous imputer une faute, à l'humanité qui nous montre que nos actes peuvent atteindre autrui parce que les actions humaines sont inextricablement emmêlées et co-solidaires. (Moreau, 2011, p. 260)

Cependant, au sein de la modernité, il est impossible de prévoir rationnellement toutes les conséquences de nos actes et le concept de regret d'agent implique alors de tenir compte des conséquences involontaires de ceux-ci et des décisions prises au moment de conflits de valeurs. Ainsi, une fois constaté le regret d'agent et les conséquences négatives de ses actes, l'agent moral tentera de les minimiser par de nouvelles actions qu'il choisit librement de faire (ou non). Didier Moreau propose alors de considérer la possibilité d'une éthique concrète pour laquelle :

il faut [...] cesser de considérer que l'éthique professionnelle est - du point de vue de l'agent moral - en rupture avec sa propre vie morale, et penser au contraire que l'enseignant tente de construire de manière cohérente sa vie personnelle. (Moreau, 2012, p. 256)

Ce concept de "vie éthique», central dans la pensée de Didier Moreau, est "une construction d'une cohérence éthique sans cesse croissante avec soi-même et ses propres émotions morales» (Moreau, 2011, p. 302) par le biais d'une liberté pratique. Cette vie éthique peut porter ses contradictions puisqu'il s'agit d'assumer les conflits de valeurs. Ainsi l'agent cherchera à agir «du mieux possible » dans une recherche de congruence et cohérence ${ }^{6}$ inatteignable dans l'absolu. 
Si l'on revient à notre analyse ci-dessus, l'obligation de congruence nous permet de conclure que tout agent moral construit une vie éthique (non restreinte seulement à l'exercice de sa profession) qui implique des postures analogues, tant dans la relation pédagogique que dans la relation à la nature. En ce sens, il est fort probable que l'agent moral finira par opter pour l'une des trois catégories que nous venons de décrire et qu'elle sera dominante dans ses pratiques, tant dans le domaine éducatif que dans sa relation à la nature. En effet, on ne saisirait pas la cohérence éthique d'une personne ayant une éthique holistique envers la nature, mais appliquant une pédagogie de guide, de contrôle en tant qu'enseignant. Mais cette analyse nous permet surtout de saisir que la relation à la nature, dès le moment où elle implique une construction éthique, pourrait, au sein du concept de vie éthique, influencer l'éthique éducative, et viceversa. Voilà qui ouvre des possibilités énormes pour la formation des enseignants, au sein de l'écoformation (formation par l'environnement et donc notamment, la nature) en particulier, et au sein de l'éducation relative à l'environnement.

Des questionnements fondamentaux me semblent alors émerger de cette conclusion. Tout d'abord, aborder la question de l'éthique environnementale peut-il représenter une ressource pour travailler indirectement les questions de l'éthique éducative? Concrètement, dans le cadre d'une approche complexe de la formation des enseignants, pourrions-nous imaginer des modules de formation éthique où les enseignants seraient amenés à réfléchir sur leur vie éthique à partir de vécus liés non seulement à leur pratique pédagogique, mais également à leur relation à la nature ? Et réciproquement les actions d'éducation relative à l'environnement pourraient-elles s'ouvrir à une réflexion sur l'influence qu'elles peuvent avoir sur la construction d'une éthique éducative ? Existe-t-il d'autres domaines alimentant la vie éthique des enseignants? D'autre part, alors que la majorité des enseignants semblent avoir construit une vie éthique à partir de l'épistémè traditionnelle du platonisme, est-il erroné de penser que ceci constitue un frein majeur dans l'application d'une réelle éducation relative à l'environnement? Ne faudrait-il pas promouvoir la compréhension des fondements éthiques présentés ci-dessus, afin de favoriser l'auto-hétéro-éco-formation des enseignants? S'ouvrir à d'autres philosophies non occidentalo-centrées peut-il représenter une opportunité dans la transition vers des épistémès éthiques adaptées aux défis environnementaux et sociétaux majeurs auxquels nous sommes confrontés aujourd'hui?

\section{BIBLIOGRAPHIE}

Afeissa H-S. (2013). Portraits de philosophes en écologistes. Paris : Éditions Dehors.

Beaudout E. (2009). Réflexion théorique sur l'existence d'un mode de pensée spécifique en éducation et formation relatives à l'environnement. Thèse de doctorat inédite. Université Paris VIII

Bolle de Bal M. (2003). Reliance, déliance, liance : émergence de trois notions sociologiques.

Sociétés 80(2), 93-99. 
Bolle de Bal M. (2009). Éthique de reliance, éthique de la reliance : une vision duelle illustrée par Edgar Morin et Michel Maffesoli. Nouvelle revue de psychosociologie, 8(2), 187-198. Récupéré le 9 décembre 2014 du site Cairn : http://www.cairn.info/revue-nouvelle-revue-depsychosociologie-2009-2-page-187.htm.

Colin L. et Le Grand J-L. (dir). (2008). L'éducation tout au long de la vie. Paris : Éditions Economica. François $1^{\mathrm{er}}$. (2015). Encyclique Laudato Si. Sobre el cuidado de la casa común. Vatican : Libreria Editrice Vaticana.

Gohier C. (2007). L'art de l'orientation de la conduite humaine en enseignement : quelle éthique et quelle déontologie ? Pour une éthique du lien. Les Sciences de l'éducation-Pour l'Ère nouvelle, 40(2), 77-93.

Hannoun H. (1998). Éduquer c'est faire un pari sur l'avenir. Dans Les valeurs en éducation et en formation. Spirale, 21, 17-24.

Hess G. (2013). Éthiques de la nature. Paris : Presses universitaires de France.

Houssaye J. (1988). Pratiques pédagogiques. Berne : Éditions Peter Lang.

Lapassade G. (1997). L'entrée dans la vie. Paris : Éditions Anthropos.

Lerbet-Séréni F. (2003). L'accompagnement entre paradoxe et quiproquo. In Accompagnement en formation d'adultes. Cahiers d'études du CUEEP, No 50-51, 203-220.

Lerbet-Séréni F. (2014). Accompagnement et paradoxes. Recherche et pratique. Communication présentée le 29 avril 2014 au Séminaire « Éducation tout au long de la vie », Université Paris VIII, laboratoire Experice, Paris, France. Récupéré le 2 mai 2014 de : http://193.54.164.31/studio8sepf/ medias/etlv2014/default1.htm

Meirieu P. (2003) L'éducation et le rôle des enseignants à l'horizon 2020. Rapport pour l'UNESCO. Récupéré le 3 avril 2015 de : http://www.meirieu.com/RAPPORTSINSTITUTIONNELS/ UNESCO2020.pdf

Moreau, D. (2011). Éducation et théorie morale. Paris : VRIN.

Moreau, D. (dir). (2012). L'éthique professionnelle des enseignants. Paris : Édition L'Harmattan.

Pineau, G. (1985). L'autoformation dans le cours de la vie : entre l'hétéro et l'écoformation. Éducation Permanente, No 78-79, 25-39

Pineau, G. (1994). Formation. Dictionnaire Encyclopédique de l'Éducation et de la Formation. Paris : Nathan.

Pineau, G. (2002). Autoformation et approche ternaire. Communication présentée au Symposium du GRAF, 9-11 mai 2002. Bordeaux, France. Récupéré le 22 août 2016 du site : http://lllearning.freeh.net/A-GRAF/Symposia/textes/2002/PineauBdx.htm.

Rabhi, P. (2010). Vers la sobriété heureuse. Paris : Éditions Actes Sud.

Rancière, J. (1987). Le maître ignorant, cinq leçons sur l'émancipation intellectuelle. Paris : Éditions Fayard.

Rousseau, J-J. (1762). Émile ou de l'éducation. Paris : Éditions Duschesne. 


\section{NOTES}

1. À noter que pour Gérald Hess, seuls les êtres humains sont capables de moralité, et donc peuvent être considérés comme des agents moraux, affirmation discutable, mais dont nous ne débattrons pas ici, par souci de concision.

2. Site de la plateforme NéoPass@ction de l'Institut français de l'Éducation : http://neo.enslyon.fr/neo

3. L'éducation tout au long de la vie est un courant de pensée au sein de l'éducation permanente qui considère une éducation toujours inachevée, intégrant toute forme d'éducation formelle, non formelle et informelle et inspirée par la philosophie du déploiement de Soi de la Bildung (Colin et Le Grand, 2008).

4. L'éthique stoïcienne consistera à trouver un équilibre entre cette tendance naturelle (hormé), qu'il faut respecter pour accéder au Bien, et la raison (logos) qui nous permet de faire des choix, au sein du Cosmos.

5. On pourra ici se référer aux travaux de Georges Lapassade (1997) qui a clairement dénoncé cette tendance à penser une éducation par rapport à un adulte-étalon.

6. La cohérence est l'adéquation à une logique générale : dire de ce que l'on fait et faire ce que l'on dit. La congruence évoque l'authenticité, l'harmonie avec soi-même : la correspondance entre intérieur et extérieur, entre ce que l'on est, pense et ressent et ce que l'on dit et fait.

\section{RÉSUMÉS}

La question de l'éthique se retrouve tant au cœur de la relation à la nature que de la relation pédagogique. C'est ce que nous tenterons de montrer dans cet article à partir de leur origine commune: la question socratique du «comment doit-on vivre?». Mais au-delà, nous analyserons comment la classification des différentes éthiques environnementales selon Gérald Hess et celle des différentes postures éducatives selon Frédérique Lerbet-Séréni, se rejoignent et coïncident avec trois grandes catégories de la pensée morale occidentale. Des convergences seront alors mises en évidence et permettront d'avancer qu'il serait pertinent de penser l'éthique professionnelle des enseignants au sein d'une vie éthique complexe, intégrant relation pédagogique et relation à la nature.

The question of ethics is at the heart of both, relationship with nature and, pedagogical relationship. This article strives to present this by building upon their common origin: the Socratic question of "how should we live?". But beyond that, we will analyze how Gerald Hess's classification of different environmental ethics and Frédérique Lerbet-Sereni's different educational positions, converge and coincide with three broad categories of Western moral thought. Convergences will then be highlighted, and allow to defend the relevancy of thinking about teachers professional ethic within a complex ethical life, integrating pedagogical relationship and relationship with nature.

El tema de la ética es central tanto en la relación con la naturaleza como en la relación pedagógica. Es lo que intentaremos demostrar en este artículo, a partir de su origen común : el questionamiento socrático del « ¿Cómo debemos vivir?». Pero más allá, analizaremos como la clasificación de las diferentes éticas ambientales según Gérald Hess y la de las diferentes posturas 
educativas según Frédérique Lerbet-Séréni, se juntan y coïnciden con tres grandes categorías del pensamiento moral occidental. Estas convergencias se evidenciarán y permitirán sugerir que sería pertinente pensar la ética profesional de los docentes, en el marco de una vida ética compleja que integre relación pedagógica y relación con la naturaleza.

\section{INDEX}

Mots-clés : éducation, environnement, éthique, relation pédagogique, éthique environnementale

Keywords : education ; environment ; ethics ; pedagogical relationship ; environmental ethics

\section{AUTEUR \\ CÉLINE CARREY}

Céline Carrey est enseignante de secondaire au lycée français de Managua (Nicaragua) depuis 12 ans. Elle est également agricultrice et mène des actions d'éducation relative à l'environnement auprès de classes de primaire visitant sa ferme caprine (www.wproject.fr/portraits/nicaragua/ celine-carrey/). Elle a obtenu un Master en Sciences de l'Éducation à l'Université de Paris VIII en 2015. Son domaine de recherche porte sur l'éthique en éducation et la relation à l'environnement. 